\title{
Esfingofilia e sistema de reprodução de Bauhinia curvula Benth. (Leguminosae: Caesalpinioideae) em cerrado no Centro-Oeste brasileiro
}

\author{
ROBERTO LOBO MUNIN ${ }^{1,2}$, REINALDO CHAVES TEIXEIRA ${ }^{1} \mathrm{e}$ \\ MARIA ROSÂNGELA SIGRIST ${ }^{1}$
}

(recebido: 10 de agosto de 2006; aceito: 18 de outubro de 2007)

\begin{abstract}
Sphingophily and breeding system in Bauhinia curvula Benth. (Leguminosae: Caesalpinioideae) in "cerrado" of Central Brazil). The reproductive biology of Bauhinia curvula was studied in a "cerrado" fragment in Mato Grosso do Sul State, Brazil. Bauhinia curvula is a shrub that blooms during six to seven months (July to November/December) and has a subterranean stem whith regeneration function. The flowers are white, hermaphrodite, zigomorphic, with nocturnal anthesis, they exhale unpleasant odor, and last about 11 hours. The stigma is wide and located above and/or in front of the anthers that presents pollen with $98.5 \%$ of viability. Nectar is produced in the hipanthium, with $26 \mu \mathrm{L}$ medium volume and solute concentration around of $15 \%$. Although flowers of B. curvula have several characteristics related to both chiropterophilous and sphingophilous syndromes, the small nectar volume and the narrow nectariferous chamber entrance seems to discourage bats visits. Bauhinia curvula is self-incompatible and depends on pollinators, therefore it did not bear fruit after spontaneous self-pollination. The studied population presented reduced reproductive effectiveness (0.07), probably due to pollen limitation. Agrius cingulatus (Sphingidae) was the only flower visitor which presents visiting behaviour, body size and proboscis length appropriated to effective pollination. This fact aid in a medium to long way the autogamic genotipe selection.
\end{abstract}

Key words - "cerrado", pollen-limitation, regeneration, sphingophily, subterranean system

RESUMO - (Esfingofilia e sistema de reprodução de Bauhinia curvula Benth. (Leguminosae: Caesalpinioideae) em cerrado no Centro-Oeste brasileiro). A biologia reprodutiva de Bauhinia curvula foi estudada em remanescente de cerrado em Mato Grosso do Sul. Bauhinia curvula é um subarbusto que floresce por seis a sete meses (junho a novembro/dezembro) e possui caule subterrâneo, espessado e gemífero, com função regenerativa. As flores são hermafroditas, zigomorfas, brancas, de antese noturna, exalam odor desagradável e duram 11 horas. O estigma é amplo e fica situado acima e/ou à frente das anteras que apresentam pólen com viabilidade de 98,5\%. Néctar é produzido no interior do hipanto, com volume médio de $26 \mu \mathrm{L}$ e concentração de solutos em torno de $15 \%$. Embora flores de B. curvula apresentem diversas características associadas às síndromes de quiropterofilia e esfingofilia, o pequeno volume de néctar e a estreita entrada da câmara nectarífera parecem não estimular visitas de morcegos. Bauhinia curvula é auto-incompatível e depende de polinizadores, pois não frutificou após autopolinização espontânea. A população estudada apresentou eficácia reprodutiva reduzida $(0,07)$, provavelmente devido à limitação de pólen. Agrius cingulatus (Sphingidae) foi o único visitante floral que apresentou comportamento de visita e comprimentos do corpo e da probóscide adequados para polinizar efetivamente as flores. Esse fato pode favorecer, a médio e longo prazo, a seleção de genótipos autogâmicos.

Palavras-chave - cerrado, esfingofilia, limitação de pólen, regeneração, sistema subterrâneo gemífero

\section{Introdução}

Bauhinia é um gênero pantropical com cerca de 300 espécies (Vaz \& Tozzi 2003), constituindo um dos maiores gêneros da subfamília Caesalpinioideae (Endress 1994). No Brasil são registradas mais de 200 espécies entre arbóreas, arbustivas e/ou lianas (Vaz \& Tozzi 2005). Podem ser utilizadas para diversos fins, como na arborização urbana, na recomposição de vegetação

1. Universidade Federal do Mato Grosso do Sul, Centro de Ciências Biológicas e da Saúde, Departamento de Biologia, Caixa Postal 549, 79070-900 Campo Grande, MS, Brasil.

2._Autor para correspondência: robertomunin@yahoo.com.br arbórea, bem como possuem interesse medicinal (Lorenzi 2000, Silva \& Cechinel Filho 2002).

As flores das espécies neotropicais do gênero exibem grande diversidade na forma, tamanho, coloração e/ou período e duração da antese (Hokche \& Ramírez 1990, Endress 1994), fato que pode ser associado ao tipo de agente polinizador. Espécies com antese diurna, tais como Bauhinia glabra Jacq., B. guianensis Aubl. e B. rutilans Spruce ex Benth, são visitadas e polinizadas por insetos (abelhas, vespas) e/ou beija-flores (Hokche \& Ramírez 1990). A polinização por visitantes noturnos, como morcegos, é registrada para seis espécies: Bauhinia brevipes Vogel. (= B. bongardii Steud.), B. cupulata Benth., B. multinervia (Kunth) D.C., B. megalandra 
Griseb., B. pauletia Pers. e B. ungulata L. (Heithaus et al. 1974, Ramírez et al. 1984, Bergallo 1990, Hokche \& Ramírez 1990, Fischer 1992, Silva et al. 1997). Como flores quiropterófilas e esfingófilas geralmente apresentam morfologia e biologia similares (Faegri \& van der Pijl 1979), essas espécies ditas quiropterófilas podem ser visitadas e/ou polinizadas por esfingídeos (e.g. B. cupulata, B. pauletia) (Heithaus et al. 1974, SilberbauerGottsberger \& Gottsberger 1975). Esfingofilia é relatada para B. cupulata e B. curvula Benth. por SilberbauerGottsberger \& Gottsberger (1975), que registram sucessivas visitas de mariposas às flores de ambas espécies. Segundo Endress (1994) Bauhinia candicans Benth. e B. platypetala Burch. ex Benth. são espécies esfingófilas. Hokche \& Ramírez (1990) mencionam esfingídeos como polinizadores noturnos de B. aculeata L. (= B. forficata Link), porém abelhas e borboletas como polinizadores diurnos.

Embora o hermafroditismo pareça ser a condição mais comum, o gênero inclui exemplos de andromonoicia (B. aculeata, B. cupulata, B. pauletia, B. ungulata), ginomonoicia (B. divaricata Lam.) e androginodioicia (B. malabarica Roxb.) (Heithaus et al. 1974, Ramírez et al. 1984, Tucker 1988, Hokche \& Ramírez 1990, Endress 1994, Silva et al. 1997). Em estudos sobre o sistema de reprodução, a auto-incompatibilidade foi verificada em B. ungulata (Bawa 1974, Ramírez et al. 1984), B. championii (Benth.) Benth. e B. corymbosa Roxb. e a autocompatibilidade em B. pauletia (Heithaus et al. 1974), B. purpurea L. e B. variegata L. (Lau et al. 2005). Esterilidade é observada em $B$. x blakeana, espécie oriunda da hibridização entre $B$. purpurea e $B$. variegata (Lau et al. 2005).

Considerando que espécies de Bauhinia com antese noturna podem potencialmente ser polinizadas por morcegos e/ou esfingídeos e que as características florais de $B$. curvula estão de acordo com as síndromes de quiropterofilia e esfingofilia, este trabalho investiga a morfologia e a biologia floral, a reprodução e os visitantes das flores desta espécie, visando esclarecer seu sistema de polinização.

\section{Material e métodos}

Este estudo foi realizado entre 2002 e 2005, em dois remanescentes contíguos de cerrado em área urbana $\left(20^{\circ} 27^{\prime} \mathrm{S}\right.$ e $54^{\circ} 37^{\prime}$ W, $530 \mathrm{~m}$ de altitude): (i) um pertencente à Reserva Particular de Patrimônio Natural da Universidade Federal de Mato Grosso do Sul (RPPN/UFMS), com 36,5 ha e vegetação constituída por cerrado e cerradão, e (ii) o outro uma área particular com 24 ha e formada principalmente por campo sujo. A região apresenta clima do tipo Tropical Chuvoso de Savana (subtipo Aw) (Köppen 1948) com inverno seco e frio (maio a agosto) e verão chuvoso e quente (dezembro a março). A precipitação média anual é de $1.532 \mathrm{~mm}$, a umidade relativa é geralmente baixa, raramente atingindo $80 \%$ e as temperaturas médias anuais variam entre 20 e $22^{\circ} \mathrm{C}$.

No campo foram anotadas informações sobre a altura das plantas, tipo e disposição das inflorescências e flores nos indivíduos, bem como a ocorrência de sistemas subterrâneos de reprodução a partir da escavação de seis plantas. Em 2002 o período de floração foi acompanhado semanalmente, de abril a outubro, sendo quantificado o número de botões e flores presentes em cinco plantas ocorrentes na borda de trilhas preexistentes na RPPN/UFMS. Estes dados foram utilizados para definir o pico de floração da espécie na área de estudo. Nos demais anos foi registrada somente a época de floração (início e término).

Dados sobre a morfologia, coloração e presença de odor nas flores foram registrados no campo e/ou laboratório. Para medir o comprimento das flores, estames e anteras foram utilizadas 34 flores. Para efeito de comparação foram feitas medidas da área e do diâmetro da entrada do hipanto (câmara nectarífera) e do volume de néctar registrado até $23 \mathrm{~h} 00 \mathrm{em}$ flores de $B$. curvula e de duas espécies quiropterófilas, $B$. holophylla (Bong.) Steud. e B. ungulata. Os eventos de biologia floral foram investigados em flores marcadas $(n=$ 20) entre $16 \mathrm{~h} 30$ e $6 \mathrm{~h} 00$. A viabilidade do pólen foi estimada pela coloração com carmim acético (Dafni 1992), a partir de 35 flores coletadas em pré-antese e provenientes de 20 plantas. Para cada flor foram quantificados 200 grãos de pólen/lâmina misturando-se os grãos de todas as anteras. A receptividade do estigma foi verificada em campo, através da presença de umidade na superfície estigmática. O número de óvulos foi quantificado em ovários de 15 flores.

$\mathrm{O}$ volume e a concentração de solutos do néctar foram quantificados em flores previamente ensacadas, através de medidas repetidas, em intervalos de duas horas, durante dois períodos: um compreendido entre 19h00-23h00 ( $n=44$ flores) e outro entre $1 \mathrm{~h} 00-5 \mathrm{~h} 00(n=14)$. Para as medidas do segundo período o néctar produzido na primeira parte da noite foi retirado e descartado às $24 \mathrm{~h} 00$. Devido à variação do volume de néctar entre as diferentes classes de horário, foi variável a quantidade de flores utilizadas para verificar a concentração de solutos entre estas classes. A concentração de solutos no néctar foi medida com auxílio de refratômetro de bolso e o volume com seringa de 1,0 mL, graduada em décimo de mililitros (Tschapka et al. 1999).

Os estudos sobre o sistema reprodutivo foram realizados em pelo menos duas estações reprodutivas e foram testados por meio de experimentos de autopolinização espontânea e manual e de polinização cruzada em flores ensacadas na préantese, emasculadas ou não, e provenientes de aproximadamente 50 indivíduos. O desenvolvimento de frutos em condições naturais (controle) foi verificado em flores marcadas e com livre acesso aos visitantes florais. A partir dos dados obtidos foram calculados o índice de autopolinização espontânea 
(IAS), o índice de auto-incompatibilidade (ISI) e a eficácia reprodutiva (ER) (Sobrevila \& Arroyo 1982) (tabela 2). Após 15 a 30 dias de maturação, os frutos obtidos nos experimentos de autopolinização manual e em condições naturais e parte dos frutos oriundos de polinização cruzada, foram coletados e abertos para a contagem das sementes.

As observações sobre os visitantes florais de B. curvula Benth. foram realizadas entre agosto e outubro nos quatro anos de estudo, principalmente no período vespertino e noturno, entre $17 \mathrm{~h} 00$ e $23 \mathrm{~h} 00$ ( 20 plantas) e $23 \mathrm{~h} 00$ e $5 \mathrm{~h} 00$ (seis plantas) durante 24 dias não consecutivos, totalizando 156 horas de observações. O comportamento dos visitantes florais de $B$. curvula foi registrado através de observações visuais diretas, sendo complementadas com auxílio de fotografias. Foram registrados os horários, o número de turnos de visitação ou visitas (período em que as flores da planta focal foram continuamente visitadas por dado visitante floral) (Fischer 1992), a freqüência de visita (= número de turnos/total de horas de observação), o tempo de permanência dos visitantes nas flores, a quantidade de flores visitadas/ turno, o recurso procurado e/ou o modo de visitar as flores. Quando possível os visitantes florais foram fotografados e/ou coletados, montados e/ou fixados em álcool 70\% e enviados a especialistas para identificação. A espessura da porção distal da língua de indivíduos de Glossophaga soricina (Pallas, 1766) da Coleção Zoológica da UFMS $(n=7)$ foi medida com auxílio de paquímetro.

Material testemunho da espécie vegetal estudada está depositado no Herbário da Universidade Federal de Mato Grosso do Sul, Campo Grande (CGMS 08828).

Nos estudos sobre o sistema reprodutivo, teste do Quiquadrado $\left(x^{2}\right)$ e Teste $t$ (Sokal \& Rohlf 1981) foram utilizados para verificar se ocorre diferença na proporção de frutos e na quantidade sementes formadas em cada tratamento, respectivamente. Teste $t$ também foi utilizado para a comparação do comprimento dos estames e anteras. ANOVA seguida de teste Tukey foram utilizados para verificar se ocorre diferença na área e diâmetro da entrada do hipanto (câmara nectarífera) e volume de néctar entre B. curvula, B. holophylla e $B$. ungulata.

\section{Resultados}

As plantas de Bauhinia curvula Benth. são subarbustos e ocorrem em porções pouco sombreadas das bordas e clareiras das áreas de estudo e atingem até 3,0 m de altura (figura 1). Nesta espécie ocorre órgão subterrâneo espessado, pouco profundo, de formato irregular, com crescimento secundário e origem caulinar (Edna Scremin Dias, dados não publicados), do qual partem os ramos aéreos da planta (figura 2) e que possui brotos responsáveis pela formação de novos ramos (Lidimila de Paula Tadei, dados não publicados). Em $B$. curvula as inflorescências são pseudo-racemos terminais (Vaz \& Tozzi 2003), com longos pedúnculos que expõem as flores acima da folhagem. Em 2002 a espécie floresceu por cerca de seis meses, com início em junho (estação seca) e término em meados de novembro (início da estação chuvosa), apresentando maior produção de botões e flores (pico de floração) em agosto (figura 5). Entretanto, a floração pode estender-se até dezembro.

As flores de B. curvula são hermafroditas, pentâmeras, zigomorfas, têm 58,2 $\pm 9,3 \mathrm{~mm}$ de comprimento e geralmente ocupam posição horizontal em relação ao solo. O cálice é reflexo com sépalas de cor castanhoesverdeada na face externa e creme a esbranquiçada na face interna (figura 3). A corola possui pétalas lineares e brancas. $\mathrm{O}$ androceu apresenta dez estames brancos, adelfos na base formando uma coluna ou tubo estaminal (Vaz \& Tozzi 2003) e heterodínamos, sendo geralmente cinco maiores (média $=49,1 \pm 2,8 \mathrm{~mm}$ ) e cinco menores (média $=37,6 \pm 2,9 \mathrm{~mm}, P<0,0001$, teste $t$ ). Os estames maiores apresentam anteras significativamente mais longas $(9,0 \pm 1,1 \mathrm{~mm})$ que os estames menores $(6,3 \pm 0,9 \mathrm{~mm}$, $P<0,0001$, teste $t)$. As anteras são rimosas, versáteis, introrsas (figura 3) e produzem pólen com viabilidade em torno de 98,5\% ( $\pm 2,1 \%)$. O estigma é capitado (Endress 1994), com superfície ampla e assimétrica e fica situado à frente e/ou acima das anteras (figura 3 ). O ovário é estipitado e possui, em média, 16 óvulos ( $\pm 2,0$ óvulos).

O néctar floral é produzido e armazenado no interior de hipanto (câmara nectarífera), que possui 55,1 $\pm 2,0$ $\mathrm{mm}^{2}$ de área (tabela 1) e é internamente revestido por tecido nectarífero. A entrada da câmara tem 1,4 $\pm 0,4$ $\mathrm{mm}$ de diâmetro e é guarnecida por tricomas (figura $13 \mathrm{~d}$ in Vaz \& Tozzi 2003). As flores produziram em média 26,0 $\mu \mathrm{L}( \pm 27,0 \mu \mathrm{L})$ de néctar, com concentração média de solutos de $15,0 \pm 2,4 \%(n=58)$. Flores de $B$. curvula apresentam volume de néctar, área e entrada da câmara nectarífera significativamente menores que flores de $B$. holophylla e B. ungulata (tabela 1).

As flores abrem no final da tarde ou início da noite, entre $18 \mathrm{~h} 00$ e $19 \mathrm{~h} 00$, e duram cerca de 11 horas. Uma a 10 flores abrem a cada noite, sendo mais comum três a quatro flores. De modo geral, há sincronia na abertura das flores dentro e entre plantas. A antese das flores inicia com o aparecimento de fendas no cálice, que infla, sendo seguido por abertura abrupta das sépalas que refletem para trás. No momento da abertura a flor exala odor forte e enjoativo similar ao de repolho, as anteras estão abertas, o estigma está receptivo e há produção de néctar (figura 6). O processo de abertura da flor demora até 30 minutos. Após a antese da flor (19h00), a produção de néctar aumenta, atinge pico às $21 \mathrm{~h} 00 \mathrm{e}$ decresce gradativamente, 

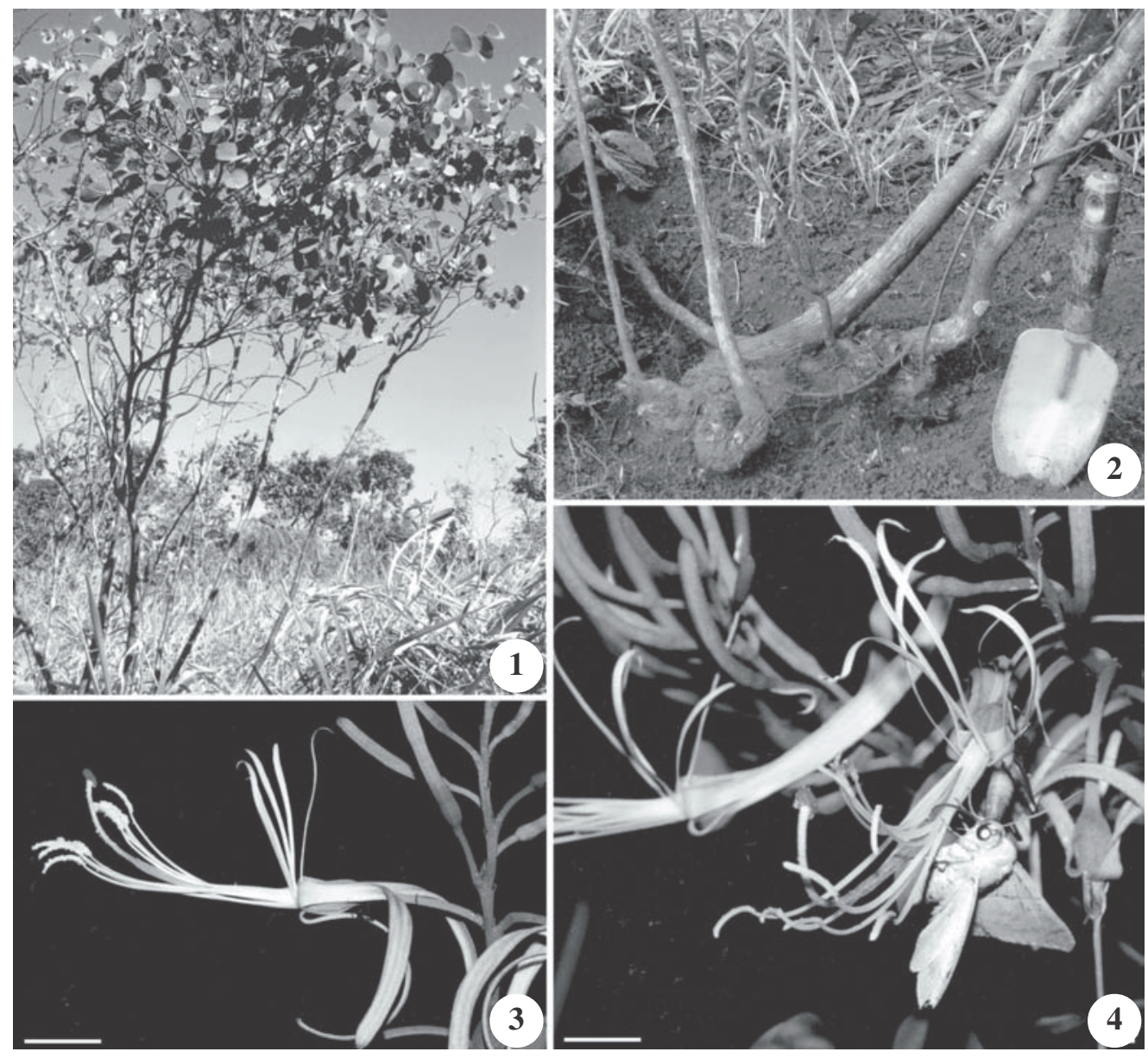

Figura 1-4. Bauhinia curvula: planta (1), caule subterrâneo (2), flor em vista lateral (3) e ao ser visitada por mariposa cf. Mimmalonidae (4). Barras $=1,0 \mathrm{~cm}$.

Figure 1-4. Bauhinia curvula: habit (1), subterranean stem (2), flower in a lateral view (3) and receiving visit from cf. Mimmalonidae moth (4). Bars $=1.0 \mathrm{~cm}$.

Tabela 1. Diâmetro da entrada da câmara nectarífera, área de produção de néctar e volume do néctar secretado por flores de Bauhinia curvula, B. holophylla e B. ungulata ocorrentes em fragmento de cerrado, Mato Grosso do Sul. Médias seguidas por letras distintas na mesma coluna diferem significativamente (Tukey, $\alpha=0,05 ; n=$ número).

Table 1. Diameter of the nectariferous chamber entrance, nectar production area and secreted floral nectar volume for Bauhinia curvula, B. holophylla and B. ungulata, that occur in a "cerrado" fragment, Mato Grosso do Sul. Averages followed by distinct letters in the same collum are significantly different (Tukey, $\alpha=0.05 ; n=$ number).

\begin{tabular}{|c|c|c|c|}
\hline \multirow[b]{2}{*}{ Espécies } & \multirow[b]{2}{*}{$\begin{array}{c}\text { Diâmetro da entrada da } \\
\text { câmara nectarífera }(\mathrm{mm}) \\
\mathrm{x} \pm s(n)\end{array}$} & \multicolumn{2}{|c|}{ Néctar } \\
\hline & & $\begin{array}{l}\text { Área de produção } \\
\qquad \begin{array}{c}\left(\mathrm{mm}^{2}\right) \\
\mathrm{X} \pm s(n)\end{array}\end{array}$ & $\begin{array}{l}\text { Volume } \\
\qquad(\mu \mathrm{L}) \\
\mathrm{X} \pm s(n)\end{array}$ \\
\hline Bauhinia curvula & $1,4 \pm 0,4(9) \quad a$ & $55,1 \pm 2,0(19) \mathrm{a}$ & $32,0 \pm 16,0(44) \mathrm{a}$ \\
\hline Bauhinia holophylla & $3,2 \pm 0,7(25) b$ & $96,8 \pm 2,4(10) b$ & $112,0 \pm 31,0(06) b$ \\
\hline Bauhinia ungulata & $3,5 \pm 0,7(14) b$ & $97,7 \pm 3,7(21) b$ & $93,0 \pm 40,0(20) b$ \\
\hline ANOVA & $\begin{array}{c}F=30,817 \mathrm{gl}=2 \\
P=0,000\end{array}$ & $\begin{array}{c}F=16,830 \mathrm{gl}=2 \\
P=0,000\end{array}$ & $\begin{array}{c}F=44,345 \mathrm{gl}=2 \\
P=0,000\end{array}$ \\
\hline
\end{tabular}


sendo que às 3h00 quase não há mais néctar (figura 6). A concentração de solutos decresce progressivamente ao longo da noite, se estabiliza às $3 \mathrm{~h} 00$, com queda abrupta às 5h00 (figura 6). As flores murcham no início da manhã por exposição ao sol e/ou pela elevação da temperatura.

$\mathrm{Na}$ população estudada não houve produção de frutos após autopolinização espontânea (IAS $=0$ ) e a taxa de frutificação após autopolinização manual foi baixa (6\%), ocasionando baixo valor no ISI (tabela 2). O percentual de frutificação em condições naturais foi baixo em relação à frutificação após polinização cruzada (ER = 0,07; tabela 2). Frutos oriundos de polinização cruzada também produziram maior número médio de sementes. Houve diferença significativa entre a proporção de frutos formados por flores autopolinizadas manualmente e aquelas submetidas à polinização cruzada $\left(x^{2}=25,190\right.$; $g l=1$ ), bem como entre o número médio de sementes produzidas entre esses dois tratamentos $(P=0,0272$; teste $t$ ). Entretanto, a proporção de frutos e o número médio de sementes produzidas por frutos formados em condições naturais em relação aos frutos autogâmicos não diferiram significativamente $\left(x^{2}=0,022 ; g l=1 ; P\right.$ $=0,3320$; teste $t$ ). Os frutos (vagens) amadurecem e abrem entre 60 e 90 dias.

Durante o período de estudo as flores foram visitadas por duas espécies de mariposas (Agrius cingulatus Fabr., Sphingidae, e por uma espécie não identificada, provavelmente pertencente a Mimallonidae, figura 4), além do besouro Cyclocephala paraguayensis Arrow. (Scarabaeidae). Agrius cingulatus visitou as flores de B. curvula entre $19 \mathrm{~h} 50 \mathrm{~min}$ e $2 \mathrm{~h} 25 \mathrm{~min}$, geralmente em noites claras e com pouco vento, sendo registrados quatro turnos de visitação em setembro de 2002 e três em outubro de 2005 (frequiência de visita $=0,045 \mathrm{~h}^{-1}$ ). Essa espécie de mariposa visitou as flores do seguinte modo: em vôo pairado defronte à flor, introduzia a probóscide distendida no interior da coluna estaminal e hipanto, provavelmente para retirar néctar. Nesse momento, contatava estigma e anteras com a porção ventral do corpo. Após cerca de 5 segundos, abandonava a flor, podendo visitar outras flores da mesma planta ou outro indivíduo próximo, ou mesmo retornar à flor visitada. A cada turno de visitação geralmente visitou até três flores. A provável espécie de Mimallonidae foi observada em 2003, 2004 e 2005, tendo sido registradas cinco visitas $\left(0,032 \mathrm{~h}^{-1}\right)$, geralmente entre $19 \mathrm{~h} 00$ e $21 \mathrm{~h} 00$. Ao visitar, a mariposa pousava geralmente sobre as sépalas, base das pétalas e/ou tubo estaminal e, muitas vezes dependurada (figura 4), introduzia a probóscide na base da flor, junto ao hipanto, para tomar néctar. Permanecia em média 1 minuto e 30 segundos nas flores visitadas e geralmente não contatava anteras e estigma.

Cerca de uma hora após a antese, besouros $C$. paraguayensis ocasionalmente agregavam-se nas flores (até seis indivíduos), permanecendo na flor provavelmente até o dia seguinte, pois durante as observações esses

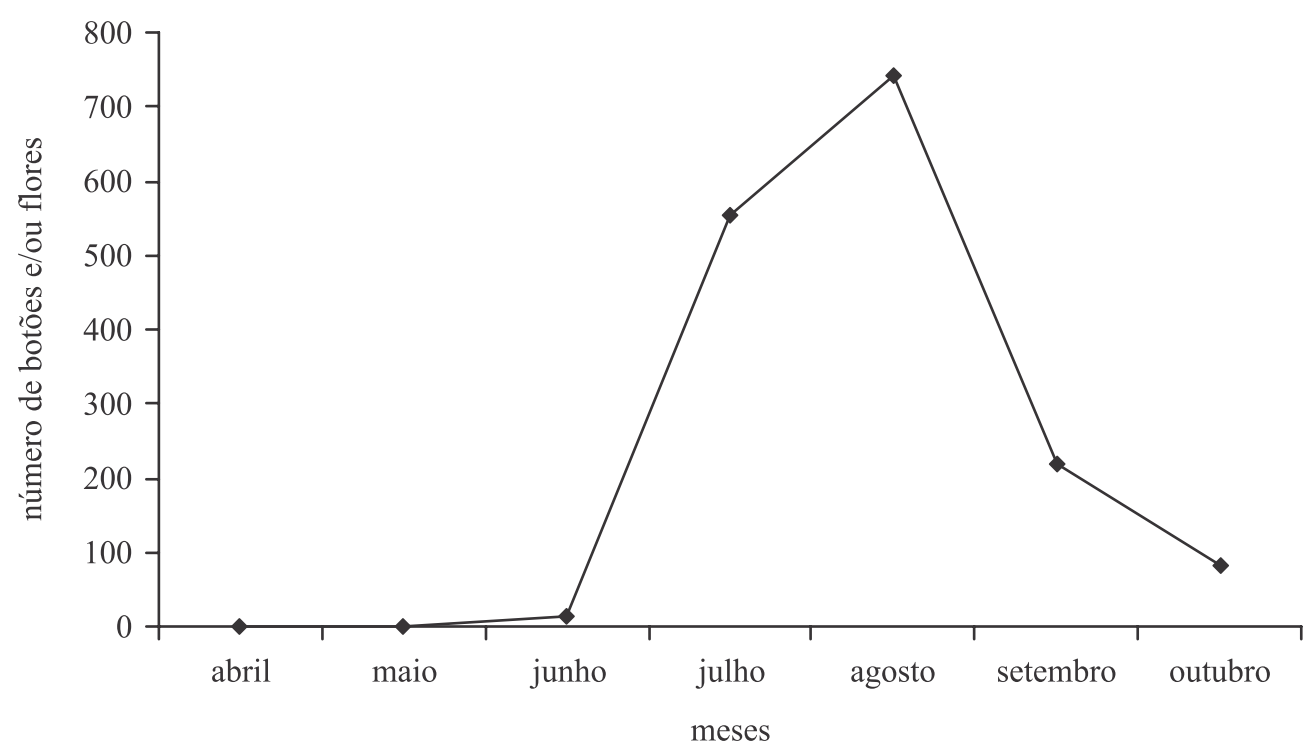

Figura 5. Fenologia de floração de Bauhinia curvula em 2002, em remanescente de cerrado, Campo Grande, Mato Grosso do Sul.

Figure 5. Blooming phenology of Bauhinia curvula in 2002, in a fragment of "cerrado", Campo Grande, Mato Grosso do Sul State. 

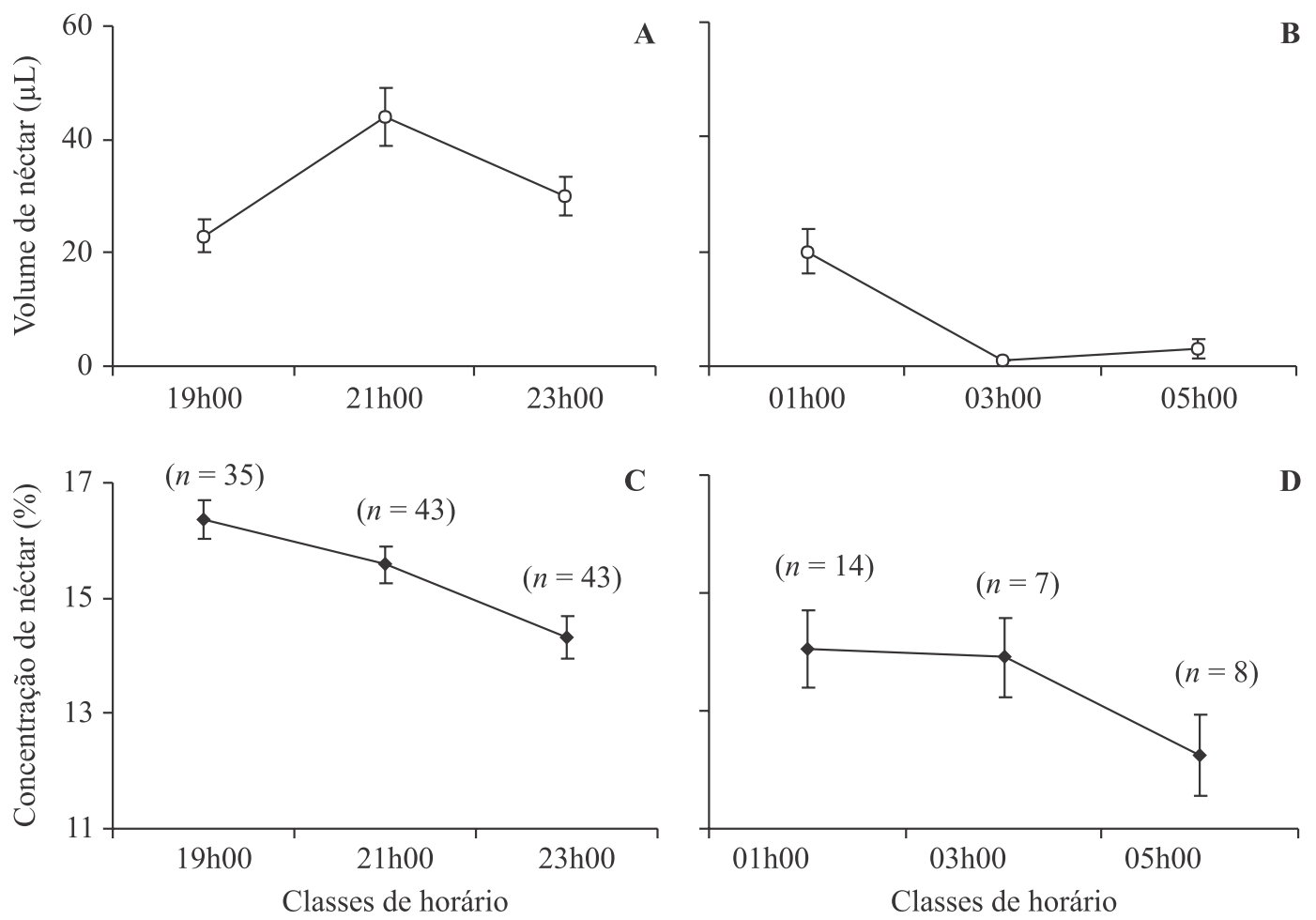

Figura 6. Volume (A e B) e concentração de solutos (C e D) no néctar floral de Bauhinia curvula em dois períodos da noite (19h00-23h00 e 01h00-05h00), em seis classes de horários, em remanescente de cerrado, Campo Grande, Mato Grosso do sul. Barras = erro padrão.

Figure 6. Volume (A and B) and solute concentration (C and D) in the floral nectar of Bauhnia curvula, in two periods of the night (19h00-23h00 and 01h00-05h00), in six hour classes, in a fragment of "cerrado", Campo Grande, Mato Grosso do Sul state. Bars = standard error.

Tabela 2. Resultados dos experimentos para testar o sistema de reprodução de Bauhinia curvula em dois remanescentes de cerrado, Campo Grande, Mato Grosso do Sul. IAS = índice de autopolinização espontânea (\% de frutos formados por flores autopolinizadas espontaneamente/\% flores autopolinizadas manualmente); ISI = índice de auto-incompatibilidade (\% de frutos formados por flores autopolinizadas manualmente/\% flores submetidas à polinização cruzada); ER = eficácia reprodutiva (\% frutos formados em condições naturais/\% frutos formados por polinização cruzada) (Sobrevila \& Arroyo 1982).

Table 2. Experiments results of the breeding system in Bauhinia curvula in two fragments of cerrado, Campo Grande, Mato Grosso do Sul. IAS = index of spontaneous self-pollination (\% of fruit set by spontaneous self-pollinated flowers/\% of hand self-pollinated flowers); ISI = index of self-incompatibility ( $\%$ of fruit set by hand self-pollinated flowers/\% of flowers submitted to crosspollination); $\mathrm{ER}=$ reproductive effectiveness (\% of fruit set in natural conditions $/ \%$ of fruit set followed crosspollination) (Sobrevila \& Arroyo 1982).

Tratamentos número de sementes $\mathrm{x} \pm s\left(\mathrm{n}^{\mathrm{o}}\right.$ frutos $)$

Autopolinização espontânea

Autopolinização manual

Polinização cruzada

Condições naturais

IAS

ISI

ER
$\%$ (no frutos/no flores)
$0 \quad(0 / 50)$
$6(3 / 50)$
$54(27 / 50)$
$4(5 / 123)$
$4,3 \pm 1,53$
$7,8 \pm 5,4$

0

0,11

0,07
$10,95 \pm 4,71(20)$ 
insetos não voavam de flor em flor. Ao pousar sobre a flor alguns besouros geralmente dirigiam-se às anteras, ingeriam pólen e, posteriormente caminhavam até a base da flor. Nessa ocasião, forçavam a entrada da coluna estaminal e atingiam o hipanto, onde permaneciam, provavelmente tomando néctar. Também utilizaram a flor como local para cópula e durante a permanência na flor eventualmente contatavam anteras e estigma. Gafanhotos (Orthoptera) não identificados foram observados com freqüência comendo diversas partes florais.

\section{Discussão}

Na população estudada de $B$. curvula Benth. o caule subterrâneo gemífero é importante na manutenção das plantas na área de estudo, pois permite a formação de novos ramos e, portanto a regeneração dos indivíduos após perda da parte aérea por seca e/ou queimadas, fato comumente observado na área de estudo (Lidimila de Paula Tadei, dados não publicados). Sistemas subterrâneos gemíferos estão relacionados à sobrevivência das espécies em condições desfavoráveis do ambiente, favorecendo a regeneração dos ramos aéreos das plantas. Aproximadamente $50 \%$ da vegetação herbácea e subarbustiva do Cerrado apresenta diversos tipos de órgãos subterrâneos espessados, sugerindo a importância ecológica dessas estruturas para espécies deste tipo de savana (Vilhalva \& Appezzatoda-Glória 2006).

Bauhinia curvula apresenta padrão de floração anual estendido (Newstrom et al. 1994), pois floresce uma vez por ano, por mais de cinco meses. Floração anual é comumente observada no gênero Bauhinia (Hokche \& Ramírez 1990) e para a maioria das espécies esfingófilas estudadas na Costa Rica (Koptur 1983, Haber \& Frankie 1989) e no cerrado do Brasil Central (Oliveira et al. 2004), embora também haja registro de espécies esfingófilas com floração contínua (Newstrom et al. 1994) (e.g. Inga brenesii Standl., Koptur 1983) ou supra-anual (e.g. Cestrum alternifolium (Jacq.) Schulz, Haber \& Frankie 1989). Entretanto, padrão estendido, com florescimento por longo período, é menos freqüente em espécies polinizadas por esfingídeos, para as quais predomina floração por breve período $(<2$ meses) ou por até quatro meses (Koptur 1983, Haber \& Frankie 1989, Oliveira et al. 2004). Para B. curvula o florescimento longo parece ser vantajoso, pois permite que a espécie floresça também na estação úmida, aumentando a possibilidade das flores serem polinizadas pelo esfingídeo Agrius cingulatus Fabr., que segundo Haber \& Frankie (1989) é sazonal, sendo registrado em floresta seca da Costa Rica somente na estação úmida.
Sazonalidade em esfingídeos foi registrada na caatinga e no brejo por Gusmão \& Creão-Duarte (2004), que discutem que a ocorrência deste grupo de lepidópteros no período chuvoso pode estar relacionada à disponibilidade de água no solo, fator importante para a passagem da fase de pupa, vivida no solo, para a fase adulta. De acordo com Haber \& Frankie (1989) e Oliveira et al. (2004) a maior concentração de esfingídeos na estação úmida ou a ocorrência em determinado local depende geralmente da presença de plantas nas quais as larvas se alimentam. Segundo Janzen (1981), larvas de esfingídeos utilizam espécies de poucas famílias botânicas (e.g. Anacardiaceae, Apocynaceae, Bignoniaceae, Euphorbiaceae, Moraceae, Rubiaceae, Solanaceae, Vitaceae), cujas folhas são ricas em alcalóides, látex, óleos essências ou outros compostos tóxicos. Esse mesmo autor (Janzen 1981) observou, em floresta tropical semidecídua, que $A$. cingulatus utilizou apenas espécies de Convolvulaceae como planta hospedeira das larvas.

As flores de B. curvula podem ser classificadas como tipo tubo (Faegri \& van der Pijl 1979), caráter comum a espécies esfingófilas (Baker 1961, Haber \& Frankie 1989, Perret et al. 2001, Johnson et al. 2004). A forma de tubo é conferida às flores pelos estames e gineceu, que participam da morfologia geral da flor (Endress 1994), sendo que o néctar fica escondido pela coluna estaminal e é acessível somente a visitantes de língua longa. Apesar de B. curvula possuir flor tipo tubo, apresenta polinização esternotríbica, pois os órgãos sexuais estão situados na porção inferior da flor e o polinizador carrega pólen na porção ventral do abdômen (Faegri \& van der Pijl 1979).

Apesar das flores de B. curvula apresentarem diversas características associadas à síndrome de quiropterofilia, tais como antese noturna, corola branca e produção de odor forte (Faegri \& van de Pijl 1979, Haber \& Frankie 1989, Proctor et al. 1996), e durarem uma noite, atributo comum à maioria das espécies polinizadas por morcegos investigadas no cerrado (Oliveira et al. 2004), consideramos esta espécie esfingófila. Isto porque o reduzido volume de néctar produzido pelas flores de B. curvula $(26 \mu \mathrm{L})$ parece desestimular visitas de morcegos, de maneira semelhante ao que foi verificado por Silva \& Sazima (1995) para a cactácea esfingófila Cereus peruvianus (L.) Miller. De modo geral flores quiropterófilas produzem volume de néctar duas a três vezes maior (e.g. Sazima et al. 1999), quando comparadas com flores esfingófilas (e.g. Haber \& Frankie 1989). A mesma relação vale para B. holophylla e B. ungulata, bem como outras espécies de Bauhinia polinizadas por morcegos ( $B$. bongardii Steud., B. pauletia Pers.) (Bergallo 1990, Hokche \& 
Ramírez 1990) e esfingídeos (B. aculeata L., Hokche \& Ramírez 1990). Além disso, a entrada mais estreita da câmara nectarífera das flores de $B$. curvula em relação às de $B$. holophylla e $B$. ungulata pode dificultar ou restringir acesso ao néctar por parte de morcegos Glossophaginae (e.g. Glossophaga soricina), que possuem língua mais espessa $(1,69 \pm 0,3 \mathrm{~mm})$ que a probóscide de mariposas Sphingidae.

O néctar de B. curvula foi quantitativamente consistente com o observado para outras flores esfingófilas (Haber \& Frankie 1989, Hokche \& Ramírez 1990, Raguso et al. 2003, Oliveira et al. 2004, Johnson et al. 2004), porém apresentou menor volume que algumas espécies esfingófilas de tubo longo como Hymenocallis littoralis (Jacq.) Salisb. (Amaryllidaceae), Lindenia rivalis Benth. (Rubiaceae) e as cactáceas Acanthocereus pentagonus (L.) Britt. \& Rose e C. peruvianus (Haber \& Frankie 1989, Silva \& Sazima 1995). O padrão de secreção de néctar de $B$. curvula concorda com o observado para Hylocereus costaricensis (Weber) Britt. \& Rose por Haber \& Frankie (1989), mas discorda da maioria das espécies esfingófilas estudadas por estes autores na Costa Rica, bem como Inga oerstediana Benth. (Koptur 1983), cujas flores apresentaram secreção contínua de néctar durante toda a noite.

A concentração média de solutos no néctar de $B$. curvula $(15,0 \%)$ foi baixa, diferindo do registrado para a maioria das espécies esfingófilas estudadas (Koptur 1983, Haber \& Frankie 1989, Silva \& Sazima 1995, Perret et al. 2001), incluindo B. aculeata (= B. forficata) na Venezuela $(19,35 \%$, Hokche \& Ramírez 1990) e no Brasil (24,5\%, variando de $17,0 \%$ a $30,0 \%$, Paulino Neto, dados não publicados). Na Costa Rica, 23 espécies polinizadas por mariposas apresentaram concentração média de solutos do néctar de 20,5\%, com variação de 5\% a 39\% (Haber \& Frankie 1989). Concentração de néctar semelhante à de $B$. curvula $(15,0 \%)$ foi registrada por Johnson et al. (2004) em Oxyanthus pyriformis (Hochst.) Skeels subsp. pyriformis (16,2\%). O padrão de concentração de solutos no néctar de $B$. curvula ao longo da noite foi semelhante ao da espécie esfingófila I. oerstediana (Koptur 1983), mais concentrado após a antese e diluído na madrugada.

Bauhinia curvula é predominantemente autoincompatível (ISI $=0,11$ ), embora possa ocorrer certo grau de compatibilidade, pois houve frutificação $(6 \%)$ após autopolinização manual, conforme também observado por Ramírez et al. (1984) em B. ungulata (8\% e 13\%). Frutificação após autopolinização não é fato raro em espécies auto-incompatíveis e pode ser importante para garantir a formação de descendentes via reprodução sexuada na escassez de polinizadores adequados. Número de sementes menor em frutos autogâmicos que frutos alogâmicos foi verificado em B. ungulata (Ramírez et al. 1984), assim como em espécies de outros grupos, por exemplo, em Swertia perennis L. (Gentianaceae) (Lienert \& Fischer 2004). A ausência de frutificação após autopolinização espontânea (IAS $=0$ ) indica que B. curvula depende de polinizadores. Nesta espécie, autopolinização espontânea é evitada provavelmente pela posição do estigma em relação às anteras (hercogamia). A hercogamia é geralmente interpretada como uma estratégia reprodutiva que promove polinização cruzada, pois evita ou restringe a autopolinização. Entretanto, de acordo com Webb \& Lloyd (1986) em espécies hermafroditas e auto-incompatíveis, a hercogamia funciona como mecanismo para evitar a obstrução da superfície estigmática com pólen inadequado (auto), além de "controlar" o comportamento do polinizador, de modo que anteras e estigma sejam contatados em uma única visita.

A reduzida eficácia reprodutiva de $B$. curvula $(0,07)$, oriunda do baixo percentual de frutificação em condições naturais, demonstra ausência ou serviço ineficiente de polinização, resultante provavelmente da baixa freqüência de visitas, incluindo a de $A$. cingulatus. Este esfingídeo foi o único visitante que apresentou comportamento de visita, comprimento do corpo (42 a $47 \mathrm{~mm}$ ) e da probóscide ( 90 a $110 \mathrm{~mm}$ ) (Haber \& Frankie 1989, Silva \& Sazima 1995) adequados para polinizar efetivamente as flores de B. curvula. Além disso, o menor número de sementes formadas em frutos de polinização aberta em relação aos de polinização cruzada mostra que B. curvula apresenta limitação de pólen (Larson \& Barret 2000), pois após polinização natural não maturaram todas as sementes que um fruto é capaz de produzir (tabela 2). Segundo Ashman et al. (2004), limitação de pólen é resultado de quantidade ou qualidade inadequada de pólen depositado no estigma, e pode ser decorrente de serviço de polinização inadequado (e.g. poucas visitas e/ou deposição de pouco pólen por visita) e deposição de pólen incompatível no estigma, respectivamente. Heithaus e colaboradores (1982) mostram que a ausência de polinização foi o principal fator na redução de sementes em $B$. ungulata na Costa Rica. Baixa frequiência de visitas de esfingídeos foi observada em algumas espécies esfingófilas e foi apontada como causa da produção limitada de frutos em condições naturais (Vesprini \& Galetto 2000, Raguso et al. 2003, Darrault \& Schlindwein 2005).

$\mathrm{Na}$ área de estudo vários fatores podem estar contribuindo para a baixa taxa de visitação de esfingídeos 
às flores de $B$. curvula, dentre eles as características do néctar, que possui baixa concentração de solutos em relação à maioria das espécies esfingófilas registrada na literatura consultada e é produzido em pequena quantidade e por período relativamente curto (oito horas). Tais características poderiam desestimular os esfingídeos, principalmente porque este grupo de insetos geralmente visita diversas espécies com flores não esfingófilas, incluindo plantas quiropterófilas (e.g. B. ungulata, tabela 2 in Haber \& Frankie 1989). Na área de estudo, esfingídeos foram observados visitando esporadicamente flores de quatro espécies quiropterófilas que ocorrem simpatricamente com B. curvula: $B$. holophylla, B. ungulata, Caryocar brasiliense Camb. e Luehea grandiflora Mart.. Destas, três espécies (exceto B. holophylla) sobrepõem parte do período de floração com B. curvula e podem ser mais "atraentes" a estes insetos, pois têm flores que produzem maior volume de néctar (e.g. B. ungulata, tabela 1). Em B. curvula, o curto período de produção de néctar também pode ser responsável pela ausência de visitantes diurnos como é comum em flores de antese noturna, incluindo espécies esfingófilas (e.g. Wolff et al. 2003). Além disso, é sabido que a fragmentação florestal promove a perda de polinizadores nativos e, consequientemente, interrompe a interação planta-polinizador (Ashman et al. 2004, Knight et al. 2006) e isto pode estar ocorrendo, pois a área de estudo corresponde a um fragmento urbano.

A mariposa cf. Mimallonidae é pilhadora do néctar floral de $B$. curvula, uma vez que não contata anteras e estigma durante suas vistas. Os besouros são pilhadores de pólen e néctar cuja presença pode desencorajar e/ou atrapalhar as visitas dos polinizadores efetivos, pois muitas vezes ficam na entrada do hipanto. Besouros Cyclocephala melanocephala Fabr. foram observados em flores de C. peruvianus (Silva \& Sazima 1995) e Datura wrightii Regel. (Raguso et al. 2003), nas quais aparentemente comiam partes florais, o que segundo Silva \& Sazima (1985) é comportamento característico da espécie. Os gafanhotos atuam de modo negativo no sucesso reprodutivo de $B$. curvula, pois reduzem a quantidade de flores por planta, que nesta espécie é pequena, geralmente 3-4 flores/noite. Segundo Heithaus et al. (1982) herbívoros que consomem flores causam grande perda no potencial reprodutivo de algumas Leguminosae. Ainda de acordo com estes autores a herbivoria floral foi um dos fatores que reduziu o potencial reprodutivo de $B$. ungulata em floresta úmida da Costa Rica.

Considerando que $B$. curvula é predominantemente auto-incompatível e depende de polinizador, que a maioria dos visitantes florais é pilhador ou herbívoro e que visitas do principal polinizador, o esfingídeo Agrius cingulatus, são pouco freqüentes, não é surpresa que na população estudada ocorra limitação de pólen. Este fato pode favorecer, a médio e longo prazo, a seleção de genótipos autogâmicos (Berjano et al. 2006). Em B. curvula, parte dos indivíduos da população é mantida a partir da rebrota do caule subterrâneo gemífero, que regenera a parte aérea após seca e/ou queimada.

Agradecimentos - Revisores anônimos pela análise do manuscrito e valiosas sugestões, Dra. Ângela Lúcia Bagnatori Sartori, Dr. Olaf Hermann Hendrik Mielke e Dr. Sergio Ide, pela identificação de Bauhinia curvula, das mariposas e do besouro, respectivamente, Dra. Edna Scremin Dias e Silvana Cristina Hammerer de Medeiros pela investigação anatômica da estrutura subterrânea.

\section{Referências bibliográficas}

ASHMAN, T.L., KNIGTH, T.M., STEET, J.A., AMARASEKARE, P., BURD, M., CAMPBELL, D.R., DUDASH, M.R., JOHNSTON, M.O., MAZER, S.J., MITCHELL, R.J., MORGAN, M.T. \& WILSON, W.G. 2004. Pollen limitation of plant reproduction: ecological and evolutionary causes and consequences. Ecology 85:2408-2421.

BAWA, K. 1974. Breeding systems of tree species of a lowland tropical community: evolutionary and ecological considerations. Evolution 28:85-92.

BAKER, H.G. 1961. The adaptations of flowering plants to nocturnal and crepuscular pollinators. Quarterly Review of Biology 36:64-73.

BERGALLO, H.G. 1990. Biologia floral e polinização de Bauhinia bongardii Steud na Serra dos Carajás, Pará. Revista Brasileira de Biologia 50:401-405.

BERJANO, R., VEGA, C., ARISTA, M. ORTIZ, P.L. \& TALAVERA, S. 2006. A multi-year study of factors affecting fruit production in Aristolochia paucinervis (Aristolochiaceae). American Journal of Botany 93: 599-606.

DAFNI, A. 1992. Pollination ecology: a practical approach. Oxford University Press, Oxford, UK.

DARRAULT, R.O. \& SCHLINDWEIN, C. 2005. Limited fruit production in Hancornia speciosa (Apocynaceae) and pollination by nocturnal and diurnal insects with long mouth parts. Biotropica 37:381-388.

ENDRESS, P.K. 1994. Diversity and evolutionary biology of tropical flowers. Cambridge University Press, Cambridge.

FAEGRI, K. \& VAN DER PIJL, L. 1979. The principles of pollination ecology. Pergamon Press, Oxford.

FISCHER, E.A. 1992. Foraging of nectarivous bats on Bauhinia ungulata. Biotropica 24:579-582. 
GUSMÃO, M.A.B. \& CREÃO-DUARTE, A.J. 2004. Diversidade e análise faunística de Sphingidae (Lepidoptera) em área de brejo e caatinga no Estado da Paraíba, Brasil. Revista Brasileira de Zoologia 21:491498.

JANZEN, D.H. 1981. Patterns of herbivory in a tropical deciduous forest. Biotropica 13:271-282.

JOHNSON, S.D., NEAL, P.R., PETER, C.I. \& EDWARDS, T.J. 2004. Fruiting failure and limited recruitment in remnant populations of the hawkmoth-pollinated tree Oxyanthus pyriformis subsp. pyriformis (Rubiaceae). Biological Conservation 120:31-39.

HABER, W.A. \& FRANKIE, G.W. 1989. A tropical moth community: Costa Rican dry forest Sphingidae. Biotropica 21:155-172.

HEITHAUS, E.R., OPLER, P.A. \& BAKER, G.H. 1974. Bat activity and pollination of Bauhinia pauletia: plantpollinator coevolution. Ecology 55:412-419.

HEITHAUS, E.R., STASHKO, E. \& ANDERSON, P.K. 1982. Cumulative effects of plant-animal interactions on seed production by Bauhinia ungulata, a neotropical legume. Ecology 63:1294-1302.

HOKCHE, O. \& RAMÍREZ, N. 1990. Pollination ecology of seven species of Bauhinia L. (Leguminosae: Caesalpinioideae). Annals of Missouri Botanical Garden 77:559-572.

KNIGHT, T.M., STEETS, J.A. \& ASHMAN, T.L. 2006. A quantitative synthesis of pollen supplementation experiments highlights the contribution of resource reallocation to estimates of pollen limitation. American Journal of Botany 93:271-277.

KÖPPEN, W. 1948. Climatologia. Fundo de Cultura Econômica, Buenos Aires.

KOPTUR, S. 1983. Flowering phenology and floral biology of Inga (Fabaceae: Mimosoideae). Systematic Botany 8:354-368.

LARSON, B.M.H. \& BARRET, S.C.H. 2000. A comparative analysis of pollen limitation in flowering plants. Biological Journal of the Linnean Society 69:503520.

LAU, C.P.Y., RAMSDEN, L. \& SAUNDERS R.M.K. 2005. Hybrid origin of "Bauhinia blakeana" (Leguminosae: Caesalpinioideae), inferred using morphological, reproductive, and molecular data. American Journal of Botany 92:525-533.

LIENERT, J. \& FISCHER, M. 2004. Experimental inbreeding reduces seed production and germination independent of fragmentation of populations of Swertia perennis. Basic and Applied Ecology 5:43-52.

LORENZI, H. 2000. Árvores brasileiras. Instituto Plantarum. Nova Odessa, v.1.

NEWSTROM, L.E., FRANKIE, G.W. \& BAKER, H.G. 1994. A new classification for plant phenology based on flowering patterns in lowland tropical rain forest trees at La Selva, Costa Rica. Biotropica 26:114-159.
OLIVEIRA, P.E, GIBBS, P.E \& BARBOSA, A.A. 2004. Moth pollination of woody species in the Cerrados of Central Brazil: a case of so much owed to so few? Plant Systematics and Evolution 245:41-54.

PERRET, M., CHAUTEMS, A., SPICHIGER, R., PEIXOTO, M. \& SAVOLAINEN, V. 2001. Nectar sugar composition in relation to pollination syndromes in Sinningieae (Gesneriaceae). Annals of Botany 87:267-273.

PROCTOR, M., YEO, P. \& LACK, A. 1996. The natural history of pollination. Harper-Collins Publishers, London.

RAGUSO, R.A., HENZEL, C., BUCHMAN, S.L. \& NABHAN, G.P. 2003. Trumpet flowers of the sonoran desert: floral biology of Peniocereus cacti and sacred Datura. International Journal of Plant Science 164:877-892.

RAMÍREZ, N., SOBREVILA, C., ENRECH, N.X. \& RUIZZAPATA, T. 1984. Floral biology and breeding system of Bauhinia benthamiana Taub. (Leguminosae), a bat pollinated tree in Venezuelan "llanos". American Journal of Botany 71:273-280.

SAZIMA, M., BUZATO, S. \& SAZIMA, I. 1999. Bat-pollinated flower assemblages and bat visitors at two Atlantic Forest sites in Brazil. Annals of Botany 83:705-712.

SILVA, K.L. \& CECHINEL FILHO, V. 2002. Plantas do gênero Bauhinia: composição química e potencial farmacológico. Química Nova 25:449-454.

SILVA, W.R. \& SAZIMA, M. 1995. Hawkmoth pollination in Cereus peruvianus, a columnar cactus of southeastern Brazil. Flora 190:339-343.

SILVA, S.S.P., PERACCHI, A.L. \& ARAGÃO, A.O. 1997. Visita de Glossophaga soricina (Pallas, 1766) às flores de Bauhinia cupulata Benth. (Leguminosae, Caesalpinioideae). Revista Brasileira de Biologia 57: 89-92.

SILBERBAUER-GOTTSBERGER, I. \& GOTTSBERGER, G. 1975. Über sphingophile Angiospermen Brasiliens. Plant Systematics and Evolution 123:157-184.

SOBREVILA, C. \& ARROYO, M.T.K. 1982. Breeding systems in montane tropical cloud forest in Venezuela. Plant Systematics and Evolution 140:19-37.

SOKAL, R.R. \& ROHLF, F.J. 1981. Biometry. W. H. Freeman, New York.

TSCHAPKA, M., VON HELVERSEN, O. \& BARTHLOTT, W. 1999. Bat pollination of Weberocereus tunilla, an epiphytic rain forest cactus with functional flagelliflory. Plant Biology 1:554-559.

TUCKER, S.C. 1988. Dioecy in Bauhinia resulting from organ suppression. American Journal of Botany 75: 1584-1597.

VAZ, A.M.S.F. \& TOZZI, A.M.G.A. 2003. Bauhinia ser. Cansenia (Leguminosae: Caesalpinioideae) no Brasil. Rodriguésia 54:55-143.

VAZ, A.M.S.F. \& TOZZI, A.M.G.A. 2005. Sinopse de Bauhinia sect. Pauletia (Cav.) DC. (Leguminosae: Caesalpinioideae: Cercideae) no Brasil. Revista Brasileira de Botânica 28:477-491. 
VESPRINI, J.L. \& GALETTO, L. 2000. The reproductive biology of Jaborosa integrifolia (Solanaceae). Why its fruits are so rare? Plant Systematics and Evolution 225:15-28.

VILHALVA, D.A.A. \& APPEZZATO-DA-GLÓRIA, B. 2006. Morfo-anatomia do sistema subterrâneo de Calea verticillata (Klatt) Pruski e Isostigma megapotamicum (Spreng.) Sherff - Asteraceae. Revista Brasileira de Botânica 29:39-47.
WEBB, C.J. \& LLOYD, D.G. 1986. The avoidance of interference between the presentation of pollen and stigmas in angiosperms. II. Herkogamy. New Zealand Journal of Botany 24:163-178.

WOLFF, D., BRAUN, M. \& LIEDE, S. 2003. Nocturnal versus diurnal pollination success in Isertia laevis (Rubiaceae): a sphingophilous plant visited by hummingbirds. Plant Biology 5:71-78. 